\title{
Erratum to: A Legal Presumption in Modernist Interpretations of Nationalism
}

\section{Unai Urrastabaso Ruiz}

\section{Erratum to:}

\section{Chapter 3 in : Modern Societies and National Identities}

The below reference at chapter-end and book-end was incorrect and it had been updated as:

Brubaker, R., \& Cooper, F. (2000). Beyond “identity". Theory and Society, 29, 1-47.

The updated original online version for this chapter can be found at DOI 10.1007/978-3-319-60077-2_3 